\title{
Adaptive Modulation for OFDM System Using Fuzzy Logic Interface
}

\author{
Seshadri K. Sastry \\ Dept.of Electronics and Communication Engineering \\ EIILM University, Sikkim \\ India
}

\section{Introduction}

Orthogonal Frequency Division Multiplexing (OFDM) is a multicarrier transmission technique, which divides the available spectrum into many carriers, each one being modulated by a low rate data stream. OFDM is a combination of modulation and multiplexing OFDM is a special case of Frequency Division Multiplexing (FDM), multiple user access is achieved by subdividing the available bandwidth into multiple channels that are then allocated to users.

However, OFDM uses the spectrum much more efficiently by spacing the channels much closer together. This is achieved by making all the carriers orthogonal to one another, preventing interference between the closely spaced carriers. Each carrier in an OFDM signal has a very narrow bandwidth (i.e. $1 \mathrm{kHz}$ ), thus the resulting symbol rate is low. This results in the signal having a high tolerance to multipath delay spread. One of the main reasons to use OFDM is to increase the robustness against frequency selective fading or narrowband interference. In a single carrier system, a single fade or interferer can cause the entire link to fail, but in a multicarrier system, only a small percentage of the subcarriers will be affected. Coded Orthogonal Frequency Division Multiplexing (COFDM) is the same as OFDM except that forward error correction is applied to the signal before transmission. This is to overcome errors in the transmission due to lost carriers from frequency selective fading, channel noise and other propagation effects.

OFDM overcomes most of the problems with both FDMA and TDMA. In FDMA many carriers are spaced apart in such a way that the signals can be received using conventional filters and demodulators. In such receivers, guard bands are introduced between carriers which results in lowering of spectrum efficiency. In OFDM sub carriers are mathematically orthogonal so that it is possible to receive signal without intercarrier interference. OFDM makes efficient use of spectrum by allowing overlap. OFDM is more resistant to frequency selective fading than single carrier systems due to dividing the channel into narrowband flat fading subchannels. OFDM eliminates ISI and IFI through use of a cyclic prefix. OFDM provides good protection against cochannel interference and impulsive parasitic noise. OFDM is less sensitive to sample timing offsets than single carrier systems (Seshadri Sastry et al., 2010 a) and (Seshadri Sastry et al., 2010 b) proposed a OFDM system with adaptive 
modulation using fuzzy logic interface to improve system capacity with maintaining good error performance. Adaptive modulation systems using ordinary hardware decision making circuits are inefficient to decide or change modulation scheme according to given conditions. Using fuzzy logic in decision making interface makes the system more efficient. The results of computer simulation show the improvement of system capacity in Rayleigh fading channel.

(Kwang et al. 2009) proposed a multi-user multiple-input multiple-output (MIMO) orthogonal frequency division multiplexing (OFDM) system with adaptive modulation and coding to improve system capacity with maintaining good error performance. The results of computer simulation show the improvement of system capacity in Rayleigh fading channel.

(Li Yanxin et al.2007) presented a novel method for demodulating the QAM signals basing on adaptive filtering. The commonly used least mean square (LMS) error adaptive filtering algorithm is employed for studying the demodulating procedure and the performance of the novel adaptive QAM demodulation. The novel adaptive QAM demodulation does not need the adaptive filter completing convergence. Therefore, the sampling rate and processing speed are decelerated. The performance of the method in theory is compared with computer simulating results. It shows that the error rates in simulation agree well with that in theory. Also, it is indicated that the demodulation method has many advantages over conventional ones, such as the powerful anti-noise ability, the small transfer delay, and the convenient implementation with DSP technology.

(Kiyoshi Hamaguchi et al.) proposed an adaptive modulation system for land mobile communications that can select one of quadrature amplitude modulation levels as a suitable modulation for propagation conditions is described. The main characteristics of the system are a mode in which information cannot be transmitted under adverse propagation conditions and a buffer memory for maintaining the data transmission rate. In the paper they confirmed that the basic performances of the adaptive modulation system using the equipment they developed and they found the measured performance was consistent with computer simulation results. Further in paper it was also confirmed that the adaptive modulation system provided a noticeable improvement in spectral efficiency and transmission quality.

Sorour Falahati, Arne Svensson, Torbjörn Ekman and Mikael Sternad proposed that when adaptive modulation is used to counter short - term fading in mobile radio channels, signaling delays create problems with outdated channel state information. The use of channel power prediction will improve the performance of the link adaptation. It is then of interest to take the quality of these predictions into account explicitly when designing an adaptive modulation scheme. They studied the optimum design of an adaptive modulation scheme based on uncoded M-QAM modulation assisted by channel prediction for the flat Rayleigh fading channel. The data rate, and in some variants the transmit power, are adapted to maximize spectral efficiency subject to average power and bit error rate constraints. The key issues studied here are how a known prediction error variance will affect the optimized transmission properties such as the SNR boundaries that determine when to apply different modulation rates, and to what extent it affects the spectral efficiency. The investigation is performed by analytical optimization of the link adaptation, using the statistical properties of a particular but efficient channel power predictor. 
Optimum solutions for the rate and transmit power are derived based on the predicted SNR and the prediction error variance.

M.K.Wasantha and W.A.C.Fernando discussed an OFDM-CDMA system with adaptive modulation schemes for future generation wireless networks. Results presented in this paper show that adaptive systems can perform better than fixed modulation based systems both in terms of BER and spectral efficiency.

\section{OFDM generation}

In OFDM we have $\mathrm{N}$ subcarriers, $\mathrm{N}$ can be anywhere from 16 to 1024 in present technology and depends on environment it is used. Block diagram of OFDM system is shown in Fig 1 below. OFDM transmitter consists of Serial to parallel converter, modulator, IFFT block, parallel to serial converter and block to add cyclic prefix. OFDM receiver consists of block to remove cyclic prefix, Serial to parallel converter, IFFT block, de modulator and parallel to serial converter

\subsection{OFDM transmitter}

Figure 1 shows the setup for a basic OFDM transmitter and receiver. An OFDM transmitter converts serial data to parallel, modulates it, converts it to time domain and transmits serial data.

\subsubsection{Serial to parallel conversion}

The input serial data stream is formatted into the word size required for transmission, e.g. 2bit/word for QPSK, and shifted into a parallel format. The data is then transmitted in parallel by assigning each data word to one carrier in the transmission.

\subsubsection{Modulator}

Modulation is a process of facilitating the transfer of information over a medium. Modulation is the process of mapping of the information on changes in carrier phase, frequency or amplitude or combination. Modulation schemes such as PSK (BPSK, QPSK, 8PSK, 16-PSK, 32-PSK) or QAM (8-QAM, 16-QAM, 32-QAM, 64-QAM) are used.

\subsubsection{Inverse fast fourier transform (IFFT)}

IFFT block is used to change domain of the signal from frequency to time. IFFT is a mathematical concept which accepts amplitudes of some sinusoids; crunch these numbers to produce time domain result. Both IFFT and FFT will produce identical result on same input

\subsubsection{Adding cyclic prefix}

Adding cyclic prefix is a process in which we extend the symbol such each symbol is more than one cycle, which allows the symbol to be out of delay spread zone and it is not corrupted. Cycli prefix will be around $10 \%-25 \%$ of the symbol time. Addition of cyclic prefix mitigates the effects of fading, intersymbol interference and increases bandwidth.. 


\subsection{Channel}

A channel model is then applied to the transmitted signal. The model allows for the signal to noise ratio, multipath, and peak power clipping to be controlled. The signal to noise ratio is set by adding a known amount of white noise to the transmitted signal. Multipath delay spread then added by simulating the delay spread using an FIR filter. The length of the FIR filter represents the maximum delay spread, while the coefficient amplitude represents the reflected signal magnitude.

\subsection{Receiver}

The receiver basically does the reverse operation to the transmitter. The guard period is removed. The FFT of each symbol is then taken to find the original transmitted spectrum. The phase angle of each transmission carrier is then evaluated and converted back to the data word by demodulating the received phase. The data words are then combined back to the same word size as the original data.

\subsubsection{Removing cyclic prefix}

Cyclic prefix added in the transmitter is removed to get perfect periodic signal

\subsubsection{FFT}

FFT block performs reverse operation to IFFT block. It is used to change domain of the signal from time to frequency

\subsubsection{De Modulator}

A demodulator performs reverse process of modulator which returns information

\subsubsection{Parallel to serial converter}

Using parallel to serial converter

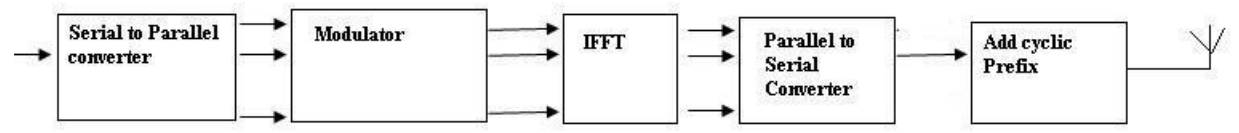

Chanuel

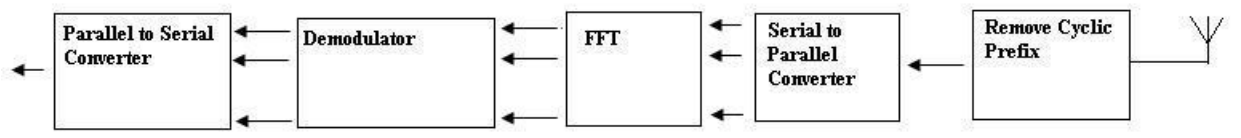

Fig. 1. OFDM system 


\section{Implementing OFDM system}

An OFDM system was modeled using Matlab to allow various parameters of the system to be varied and tested. The aim of doing the simulations was to measure the performance of OFDM under different channel conditions, and to allow for different OFDM configurations to be tested. The main criterion of this chapter is to compare performance of Fuzzy logic based adaptive modulated OFDM system with adaptive modulated OFDM system. Matlab program to implement OFDM system and simulation results are given below

\subsection{Matlab program to implement OFDM system}

\subsubsection{Transmitter design}

\subsubsection{Defining parameters}

$\mathrm{M}=16 ; \%$ Size of signal constellation

$\mathrm{k}=\log 2(\mathrm{M}) ; \%$ Number of bits per symbol

$\mathrm{n}=3 \mathrm{e} 4 ; \%$ Number of bits to process

nsamp $=1 ; \%$ Oversampling rate

\subsubsection{Signal source}

t_data $=$ randint $(9600,1) ; \%$ Random binary data stream

$\%$ Plot first 40 bits in a stem plot.

stem(t_data(1:40),'filled');

title('Random Bits');

xlabel('Bit Index'); ylabel('Binary Value');

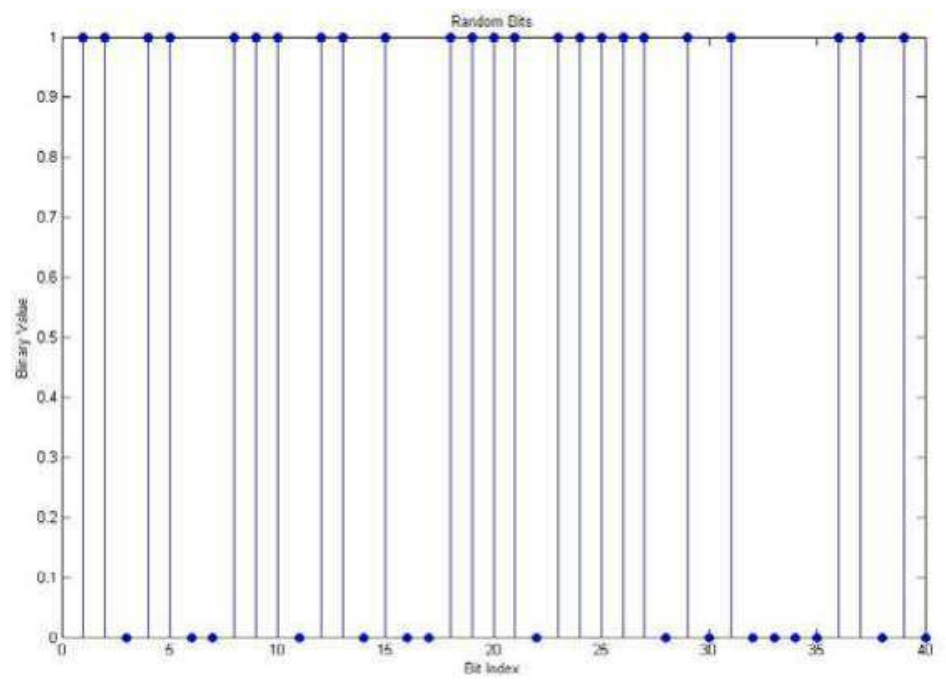

Fig. 2. Random Bits stream (Used by signal source)

xsym = bi2de(reshape(t_data,k,length(t_data)/k).','left-msb'); 
figure;

$\operatorname{stem}(\operatorname{xsym}(1: 10))$;

title('Random Symbols');

xlabel('Symbol Index'); ylabel('Integer Value');

close all

clear all

clc

$\mathrm{M}=16$; \% Size of signal constellation

$\mathrm{k}=\log 2(\mathrm{M}) ; \%$ Number of bits per symbol

t_data=randint $(9600,1)$;

stem(t_data(1:40),'filled');

title('Random Bits');

xlabel('Bit Index'); ylabel('Binary Value');

$\mathrm{ti}=(0: 1: 49)$;

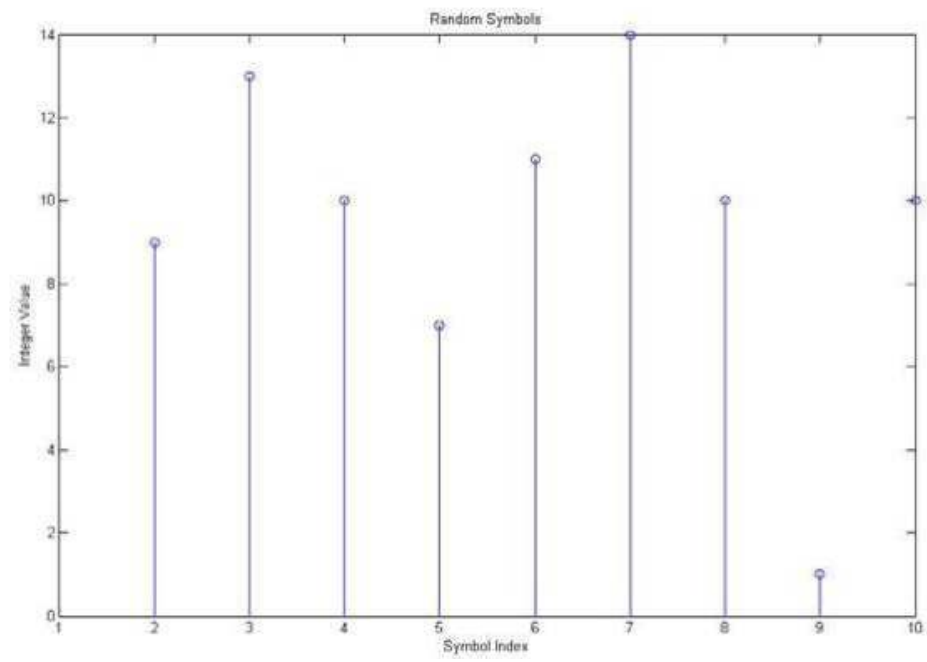

Fig. 3. Bits converted to symbols

xsym = bi2de(reshape(t_data,k,length(t_data)/k).','left-msb');

$\% \%$ Stem Plot of Symbols

$\%$ Plot first 10 symbols in a stem plot.

figure; \% Create new figure window.

stem(xsym(1:10));

title('Random Symbols');

xlabel('Symbol Index'); ylabel('Integer Value');

$\mathrm{x}=1$;

$\mathrm{si}=1$;

for $d=1: 100$;

data $=\mathrm{t} \_$data $(\mathrm{x}: \mathrm{x}+95)$;

$\mathrm{x}=\mathrm{x}+96$;

$\mathrm{k}=3$; 
$\mathrm{n}=6$;

s1=size(data,2); \% Size of input matrix $\mathrm{j}=\mathrm{s} 1 / \mathrm{k}$;

\subsubsection{Convolutional encoding}

constlen $=7$;

codegen $=[171$ 133]; \% Polynomial

trellis = poly2trellis (constlen, codegen);

codedata = convenc(data, trellis);

\subsubsection{Interleaving data}

s2=size (codedata,2);

$\mathrm{j}=\mathrm{s} 2 / 4$;

matrix=reshape(codedata,, 4$)$;

intlvddata $=$ matintrlv $\left(\text { matrix }^{\prime}, 2,2\right)^{\prime} ; \%$ Interleave.

intlvddata=intlvddata';

dec=bi2de(intlvddata','left-msb');

\subsubsection{16-QAM modulation}

$\mathrm{M}=16$;

$\mathrm{y}=\operatorname{qammod}(\mathrm{dec}, \mathrm{M})$;

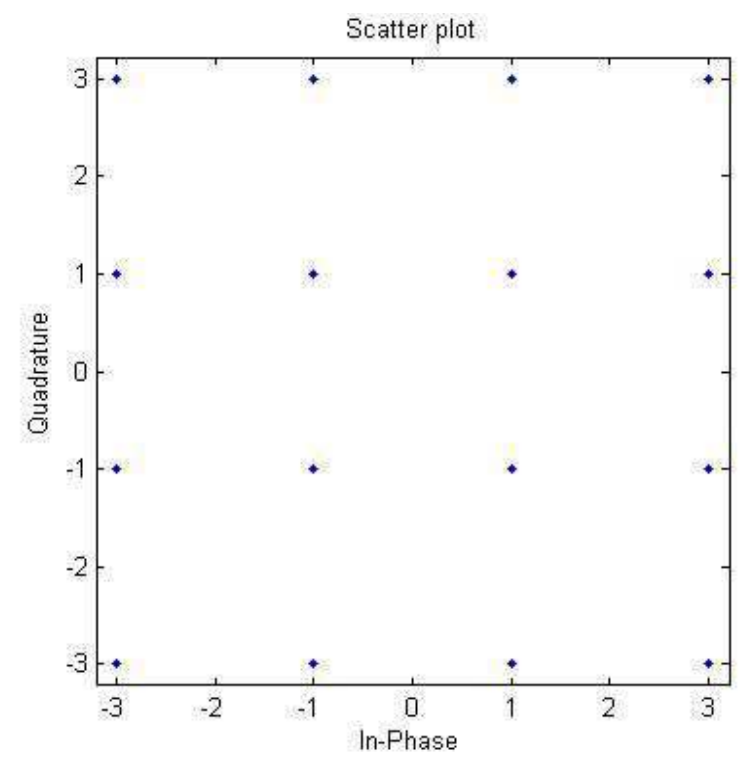

Fig. 4. Constellation ordering of 16-QAM modulator

\subsubsection{Pilot insertion}

lendata=length $(\mathrm{y})$;

pilt=3+3j; 
nofpits=4;

$\mathrm{k}=1$;

for $\mathrm{i}=(1: 13: 52)$

pilt_data1 $(\mathrm{i})=$ pilt;

for $\mathrm{j}=(\mathrm{i}+1: \mathrm{i}+12)$;

pilt_data $1(\mathrm{j})=\mathrm{y}(\mathrm{k})$;

$\mathrm{k}=\mathrm{k}+1$;

end

end

pilt_data1=pilt_data1'; $\%$ size of pilt_data $=52$

pilt_data(1:52)=pilt_data1(1:52); \% upsizing to 64

pilt_data(13:64)=pilt_data1(1:52); \% upsizing to 64

for $i=1: 52$

pilt_data(i+6)=pilt_data1(i);

end

3.8 IFFT

ifft_sig=ifft(pilt_data',64);

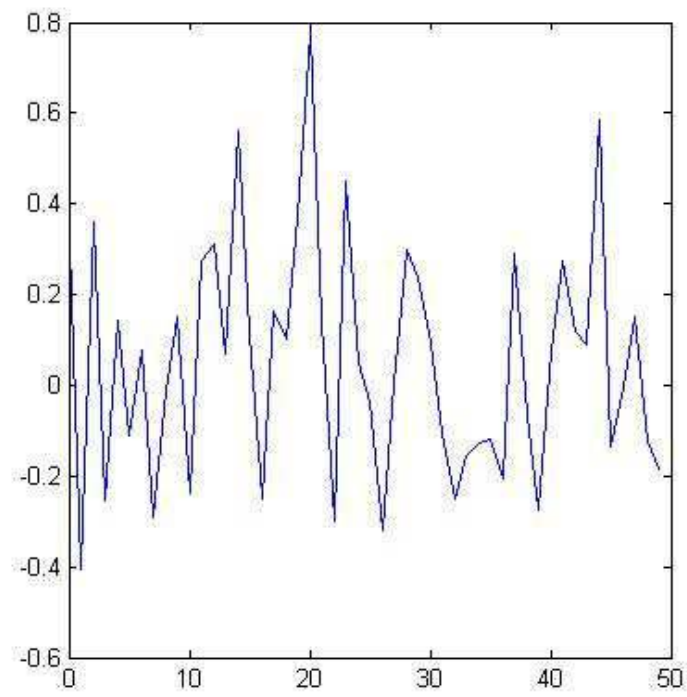

Fig. 5. OFDM Signal

\subsubsection{Adding cyclic extension}

cext_data=zeros $(80,1)$;

cext_data(1:16)=ifft_sig(49:64);

for $i=1: 64$

cext_data $(i+16)=i f f t \_s i g(i)$;

end 


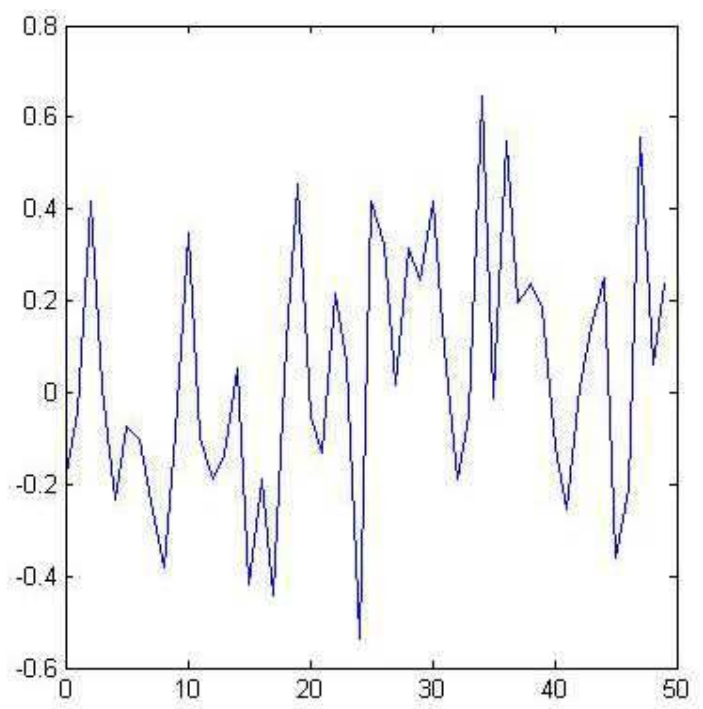

Fig. 6. OFDM Signal after Cyclic prefix

\subsubsection{Channel}

$\mathrm{o}=1$;

for $\mathrm{snr}=0: 2: 50$

ofdm_sig=awgn(cext_data,snr,'measured'); \% Adding white Gaussian Noise

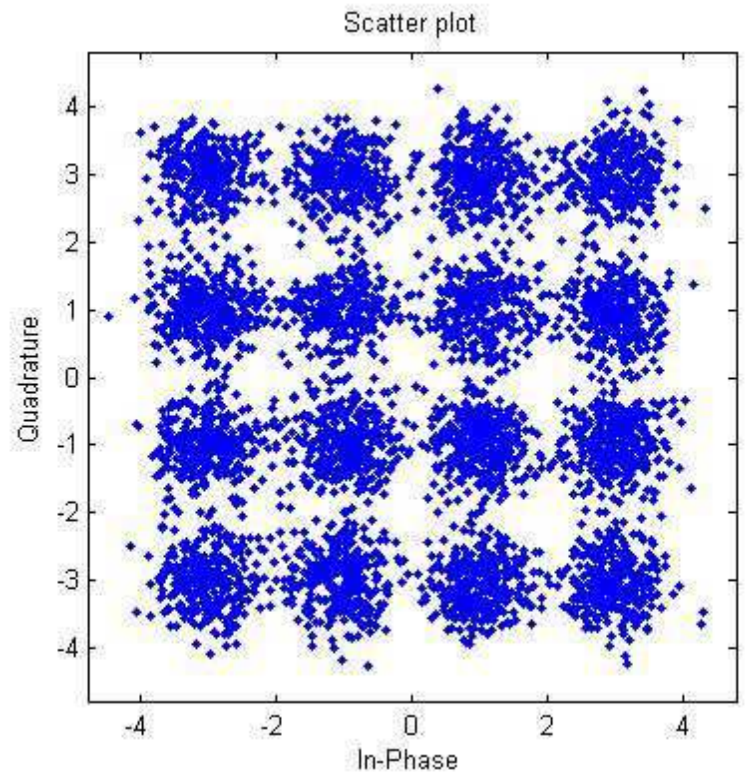

Fig. 7. Constellation ordering at receiver 


\subsubsection{Receiver design}

\subsubsection{Removing cyclic extension}

for $i=1: 64$

rxed_sig(i)=ofdm_sig(i+16);

end

$\%$ FFT

ff_sig=fft(rxed_sig,64);

$\%$ Pilot Synch

for $i=1: 52$

synched_sig1(i)=ff_sig $(\mathrm{i}+6)$;

end

$\mathrm{k}=1$;

for $\mathrm{i}=(1: 13: 52)$

for $\mathrm{j}=(\mathrm{i}+1: \mathrm{i}+12)$;

synched_sig $(\mathrm{k})=$ synched_sig1(j);

$\mathrm{k}=\mathrm{k}+1$;

end

end

\subsubsection{Demodulation}

dem_data= qamdemod(synched_sig,16);

bin=de2bi(dem_data','left-msb');

bin=bin';

\subsubsection{De-interleaving}

deintlvddata $=$ matdeintrlv $($ bin,2,2); \% De-Interleave

deintlvddata=deintlvddata';

deintlvddata=deintlvddata $(:)^{\prime}$;

\subsubsection{Decoding data}

$\mathrm{n}=6$;

$\mathrm{k}=3$;

decodedata $=$ vitdec(deintlvddata,trellis,5,'trunc','hard'); \% decoding datausing veterbi decoder

rxed_data=decodedata;

\subsubsection{Calculating BER}

rxed_data=rxed_data(:)';

errors $=0$;

c=xor(data,rxed_data);

errors $=n n z(c)$;

$\mathrm{BER}(\mathrm{si}, \mathrm{o})=$ errors/length(data);

$\mathrm{O}=\mathrm{O}+1$;

end $\mathrm{si}=\mathrm{si}+1$;

end for $\mathrm{col}=1: 25$;

$\operatorname{ber}(1, \mathrm{col})=0$; 
for row $=1: 100$;

$\operatorname{ber}(1, \mathrm{col})=\operatorname{ber}(1, \mathrm{col})+\mathrm{BER}(\mathrm{row}, \mathrm{col})$;

end

end

ber=ber./100;

$\% \%$

figure

$\mathrm{i}=0: 1: 49$;

semilogy(i,ber);

title('BER vs SNR');

ylabel('BER');

xlabel('SNR (dB)');

grid on

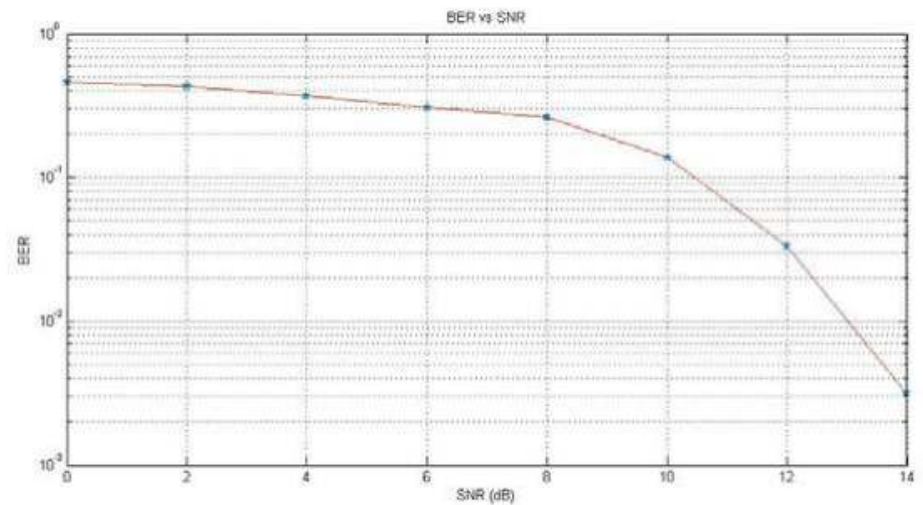

Fig. 8. Bit error rate of OFDM system

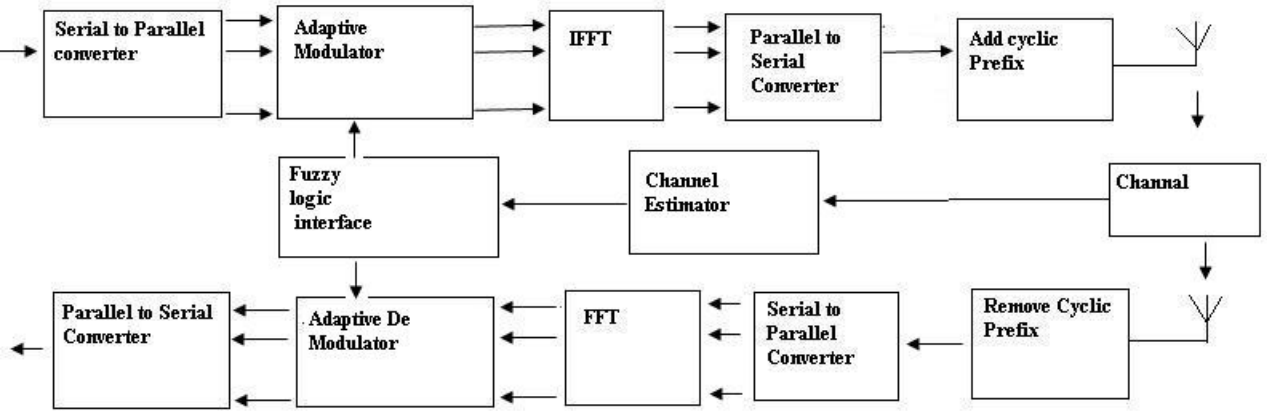

Fig. 9. Adaptive modulated OFDM system

\section{Adaptive modulation for OFDM system using fuzzy logic interface}

In a OFDM system using lower order modulators such asBPSK, 4 QAM and 8 QAM will improve Bit Error Rate (BER) but decreases spectral efficiency and speed, on the other hand employing higher order modulators such as 64 QAM, 128 QAM, 256QAM and 512 QAM will 
increase spectral efficieny and speed but result in poor BER. So to achieve good trade-off between spectral efficiency and overall BER (Bit Error Rate). Adaptive modulation is used.

\subsection{Adaptive modulator and demodulator}

At the transmitter the adaptive modulator block consists of different modulators which are used to provide different modulation orders. The switching between these modulators will depend on the instantaneous SNR. The goal of adaptive modulation is to choose the appropriate modulation mode for transmission depending on instantaneous SNR, in order to achieve good trade-off between spectral efficiency and overall BER. Adaptive modulation is a powerful technique for maximizing the data throughput of subcarriers allocated to a user. Adaptive modulation involves measuring the SNR of each subcarrier in the transmission, then selecting a modulation scheme that will maximize the spectral efficiency, while maintaining an acceptable BER.

\subsection{Program to implement adaptive modulation for OFDM}

\subsubsection{Defining parameters}

clear

$\mathrm{N}=256$;

$\mathrm{P}=256 / 8$;

$\mathrm{S}=\mathrm{N}-\mathrm{P}$;

$\mathrm{GI}=\mathrm{N} / 4$;

$\mathrm{M}=2$;

pilotInterval $=8$;

$\mathrm{L}=16$;

nIteration $=500$;

SNR_V = [0:1:34];

ber = zeros $(1$,length(SNR_V));

Ip $=$ [1:pilotInterval:N];

Is = setxor $(1: \mathrm{N}, \mathrm{Ip})$;

$\mathrm{Ep}=2$;

\subsection{2 fft}

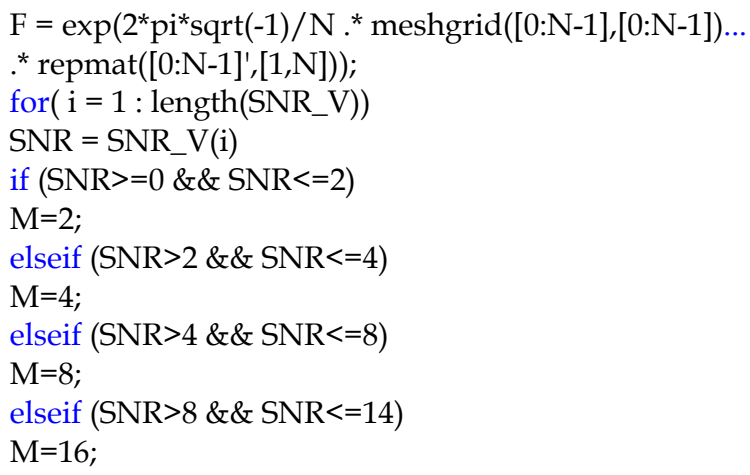


elseif (SNR $>14 \& \&$ SNR $<=20)$

$\mathrm{M}=32$;

elseif (SNR>20 \&\& SNR<=27)

$\mathrm{M}=64$;

elseif (SNR $>27$ \&\& SNR<=34)

$\mathrm{M}=128$;

end

for $(\mathrm{k}=1$ : nIteration $)$

$\mathrm{h}(1: \mathrm{L}, 1)=\operatorname{random}($ 'Normal', $0,1, \mathrm{~L}, 1)+\ldots$

$\mathrm{j}$ * random('Normal',0,1,L,1);

$\mathrm{h}=\mathrm{h} . / \operatorname{sum}(\operatorname{abs}(\mathrm{h}))$;

\subsubsection{Transmission of data}

TrDataBit = randint $(\mathrm{N}, 1, \mathrm{M})$;

TrDataMod = qammod $($ TrDataBit,$M)$;

$\operatorname{TrDataMod}(\mathrm{Ip})=\mathrm{Ep}$ * TrDataMod(Ip);

TrDataIfft $=$ ifft $($ TrDataMod, $N)$;

TrDatalfftGi = [TrDataIfft $(\mathrm{N}-\mathrm{GI}+1: \mathrm{N})$; TrDataIfft $]$;

TxDataIfftGi = filter(h,1,TrDataIfftGi);

TxDataIfftGiNoise $=\operatorname{awgn}($ TxDataIfftGi ...

, SNR - db(std(TxDataIfftGi)));

TxDataIfft $=$ TxDataIfftGiNoise $(\mathrm{GI}+1: \mathrm{N}+\mathrm{GI})$;

TxDataMod $=\mathrm{fft}(\mathrm{T} \times$ DataIfft,N $)$;

\subsubsection{Channel estimation}

Spilot $=$ TrDataMod(Ip);

Ypilot = TxDataMod(Ip);

$\mathrm{G}=(\mathrm{Ep} \text { * length }(\mathrm{Ip}))^{\wedge}-1 \ldots$

${ }^{*}$ ctranspose $\left(\operatorname{sqrt}(\mathrm{Ep})^{*} \operatorname{diag}\left(\right.\right.$ Spilot) ${ }^{*}$ ctranspose $\left.(\mathrm{F}(1: \mathrm{L}, \mathrm{Ip}))\right)$;

hHat $=\mathrm{G}^{*}$ Ypilot;

TxDataBit $=$ qamdemod $($ TxDataMod. $/(\mathrm{fft}(\mathrm{hHat}, \mathrm{N})), \mathrm{M})$;

\subsubsection{Bit error rate computation}

$[\mathrm{nErr}$ bErr(i,k)] = symerr(TxDataBit(Is),TrDataBit(Is));

end

end

f1 = figure(1);

set(f1,'color',[1 111$]$ );

semilogy(SNR_V,mean(bErr'),'r-d')

xlabel('SNR ');

ylabel('BER')

grid on;

hold on; 


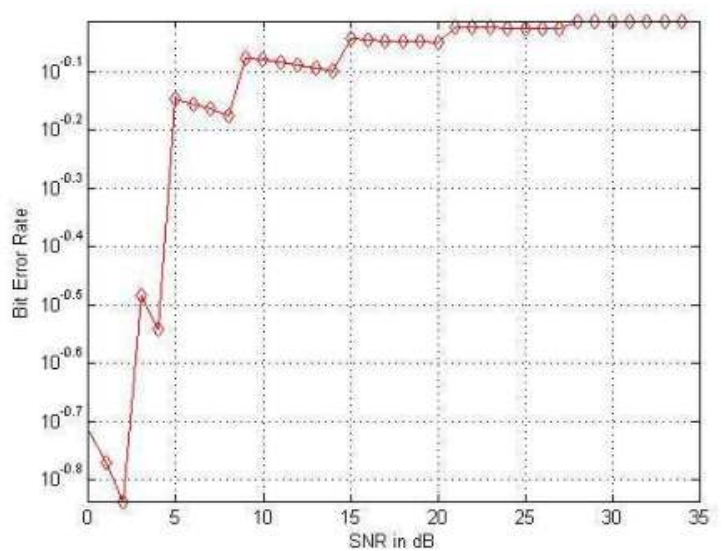

Fig. 10. Bit Error Rate of Adaptive modulated OFDM System

\section{FIS (Fuzzy Interface system)}

FIS (Fuzzy Interface system) is the decision making system in Channel Estimator (SNR estimator) used in adaptive modulation. It is modeled in Matlab 7.4 Fuzzy Interface editor. It takes instantaneous SNR (Signal to Noise Ratio) and Present modulation order as inputs and controls the modulation order of modulator and demodulator blocks.

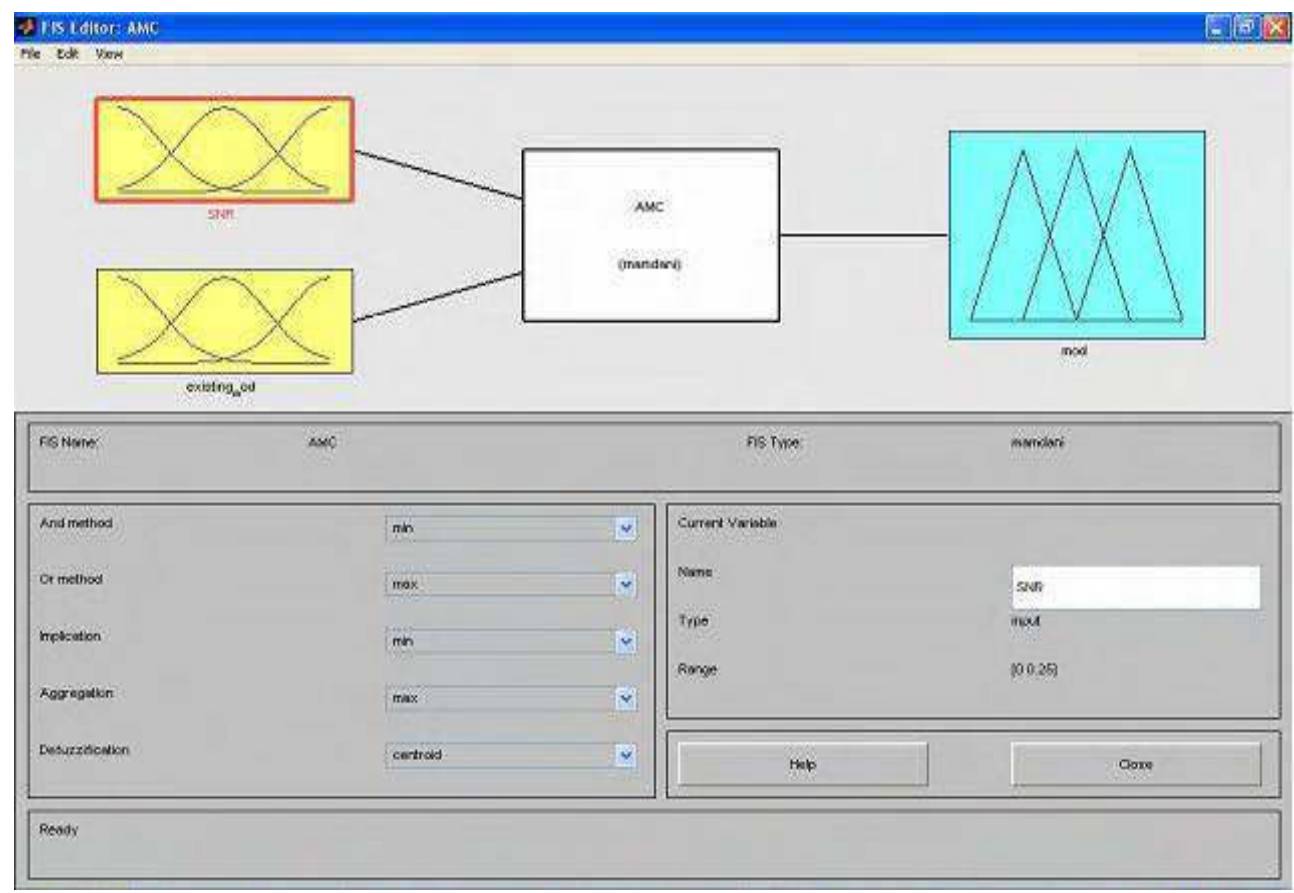

Fig. 11. FIS system 
FIS (Fuzzy Interface System) consists of two inputs and one output as shown in figure above Input one SNR and input two present mod. The membership function of SNR and present mod is shown in figures 12 and 13. For SNR, three membership functions are taken namely low_SNR, medium_SNR and high_SNR. For present modulation six membership functions are taken namely QPSK, 8QAM, 16QAM, 32QAM, and 64QAM and128QAM.

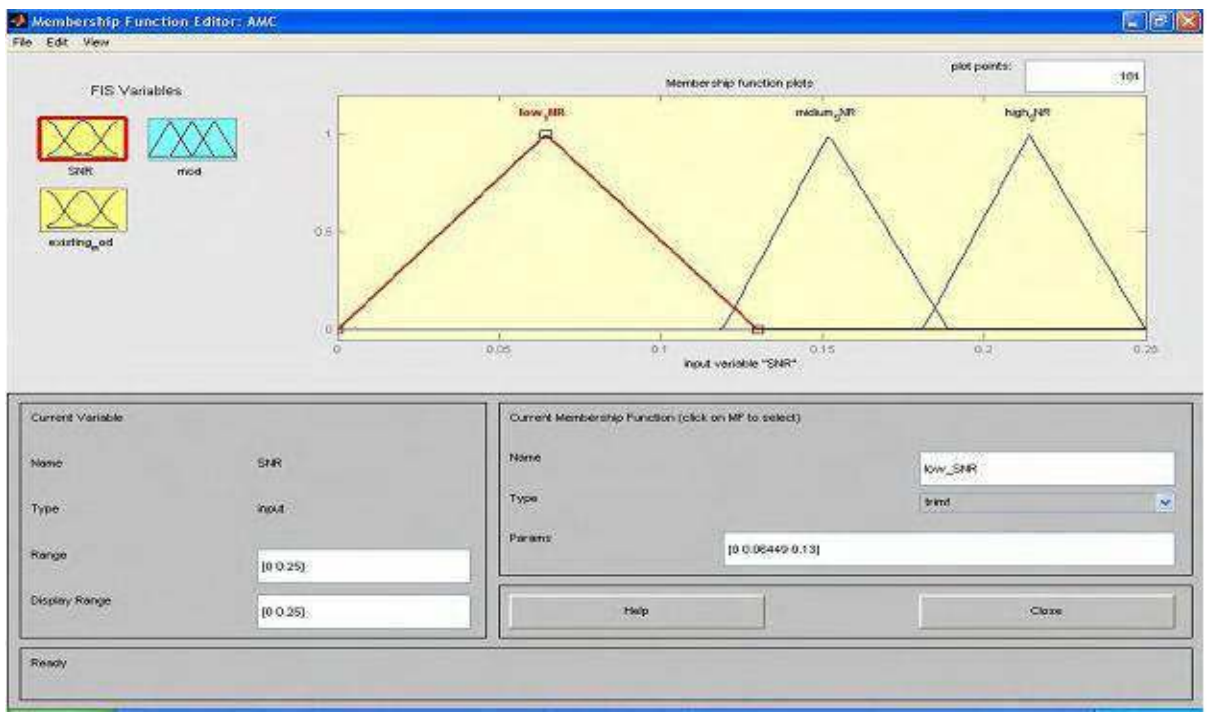

Fig. 12. SNR membership functions

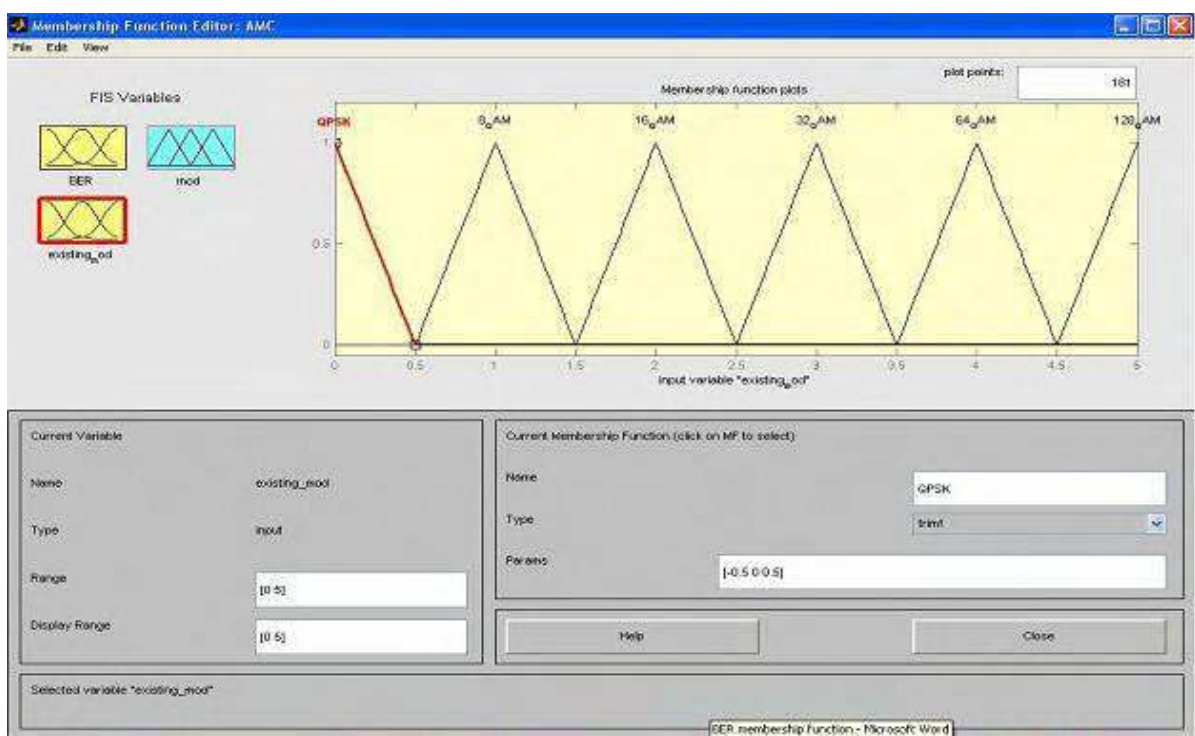

Fig. 13. Present mod membership functions 


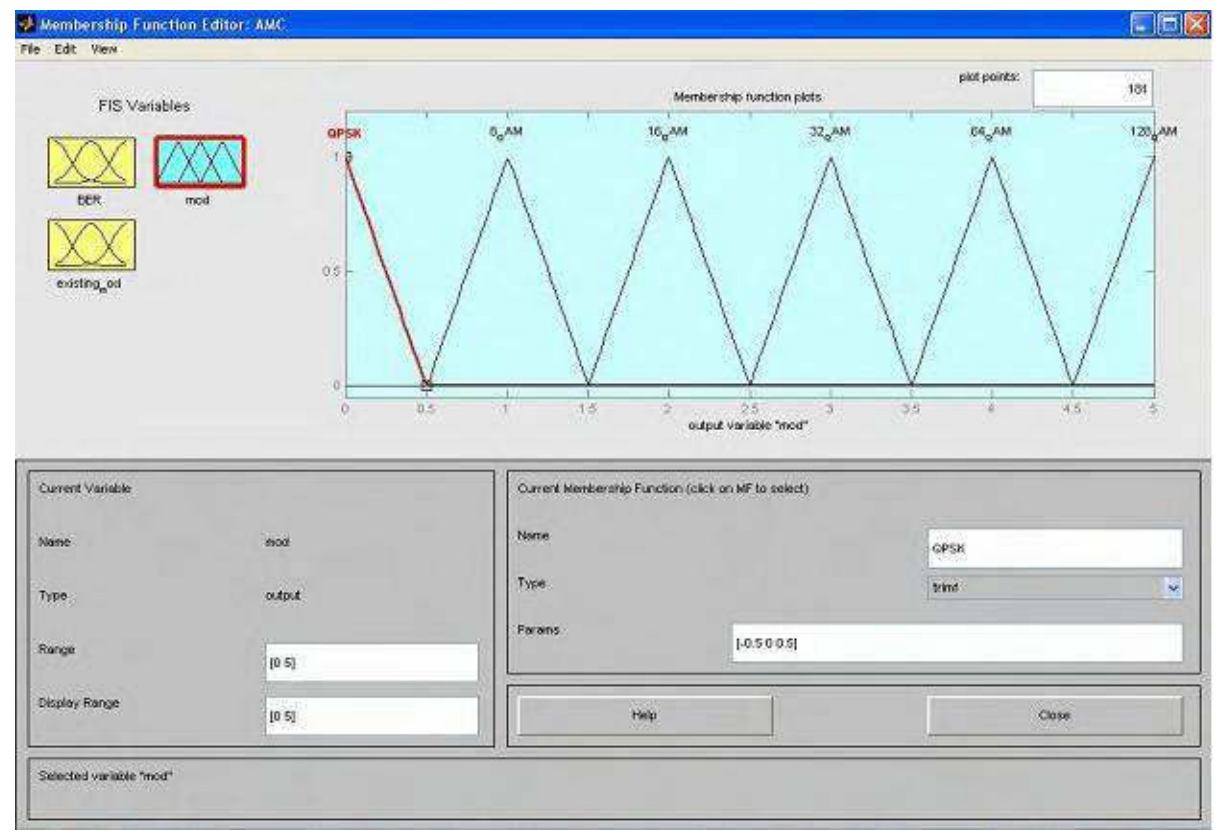

Fig. 14. Output membership function

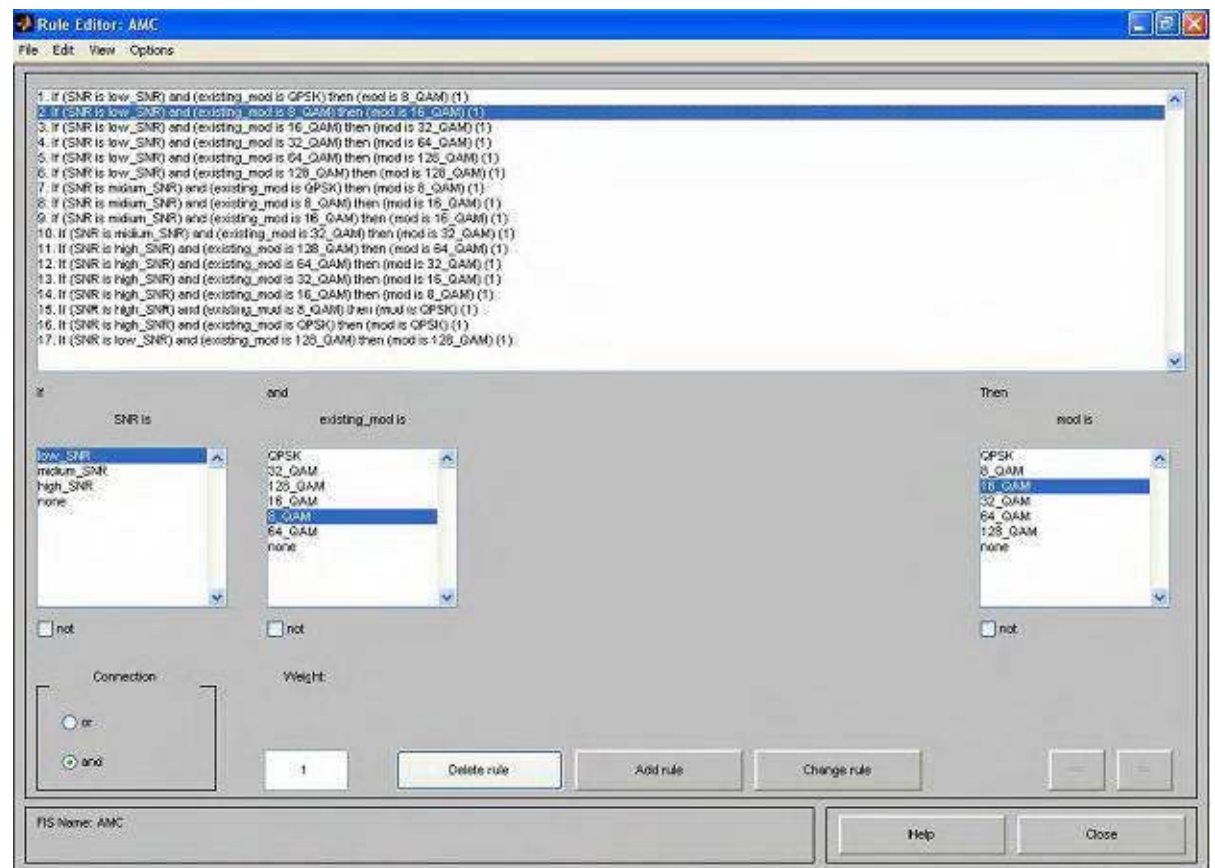

Fig. 15. Rules editor 
Rules are edited in rules editor which gives conditions to select modulation order depending on channel estimation (SNR). Example for rules are as follows, if BER is low increase the modulation order by one level compared to present modulation order, if BER is high then decrease the modulation order by one compared to present modulation order. If BER is average then increase the modulation order by one compared to present modulation order.

The above proposed system was simulated in Matlab7.4., Using fuzzy logic in decision making is a good choice because ordinary (non fuzzy) system is controlled by plain if and else statements, for example, if for poor SNR (Signal To Noise Ratio) range is declared as 0 to 4 , if input is 4.1 then the input is not considered as poor SNR (But it is poor). If we use fuzzy logic in above case 4.1 is also considered as poor SNR. So using FIS (Fuzzy interface system) increases the performance adaptive modulation system.

Fig 16 shows the output of FIS (fuzzy interface system) for given set of inputs, output is selected based on given rules. Bit Error Rate performance of the simulated system is shown in Fig 17.

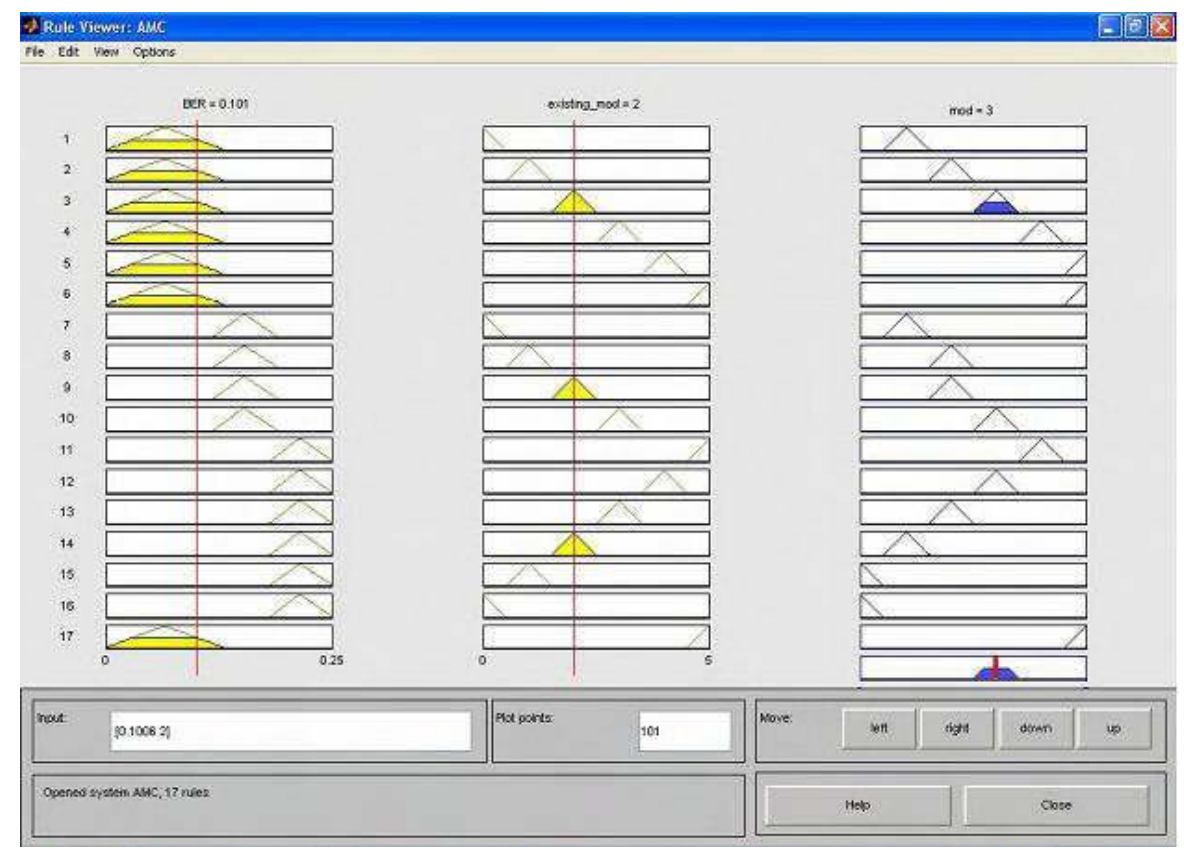

Fig. 16. Simulation result

Fig 17 shows comparison bit error rates of adaptive modulated OFDM system and fixed modulated OFDM system. Fig 18 gives plot showing the comparison of performances of adaptive modulated OFDM system using fuzzy logic and adaptive modulated OFDM system using ordinary control logic. It was shown that OFDM system using Fuzzy logic performs better than OFDM system using ordinary control logic. Using FIS (Fuzzy interface system) in implementing adaptive modulation for OFDM system increases performance of system since it responds to channel condition and maintains good performance (Bit Error Rate) and capacity (spectral efficiency) efficiently than system using ordinary control logic. 


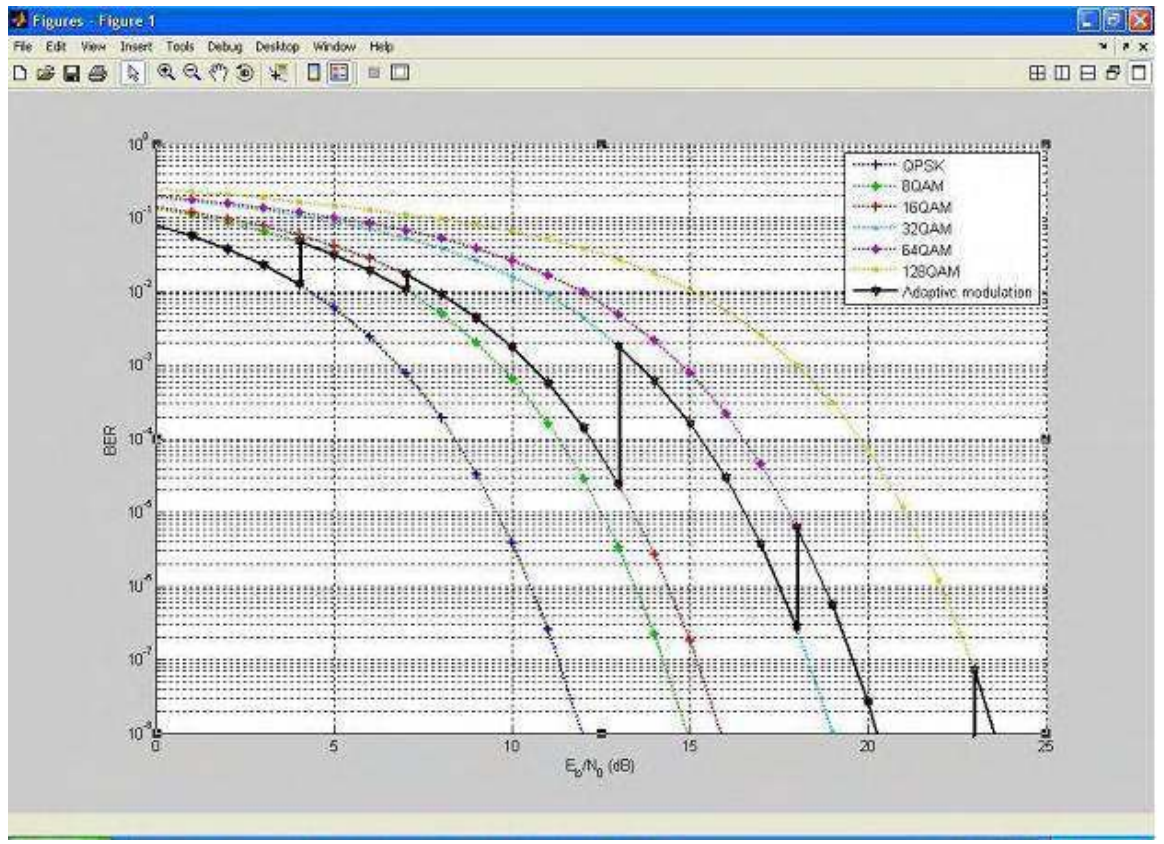

Fig. 17. BER comparison of proposed scheme and fixed modulation schemes.

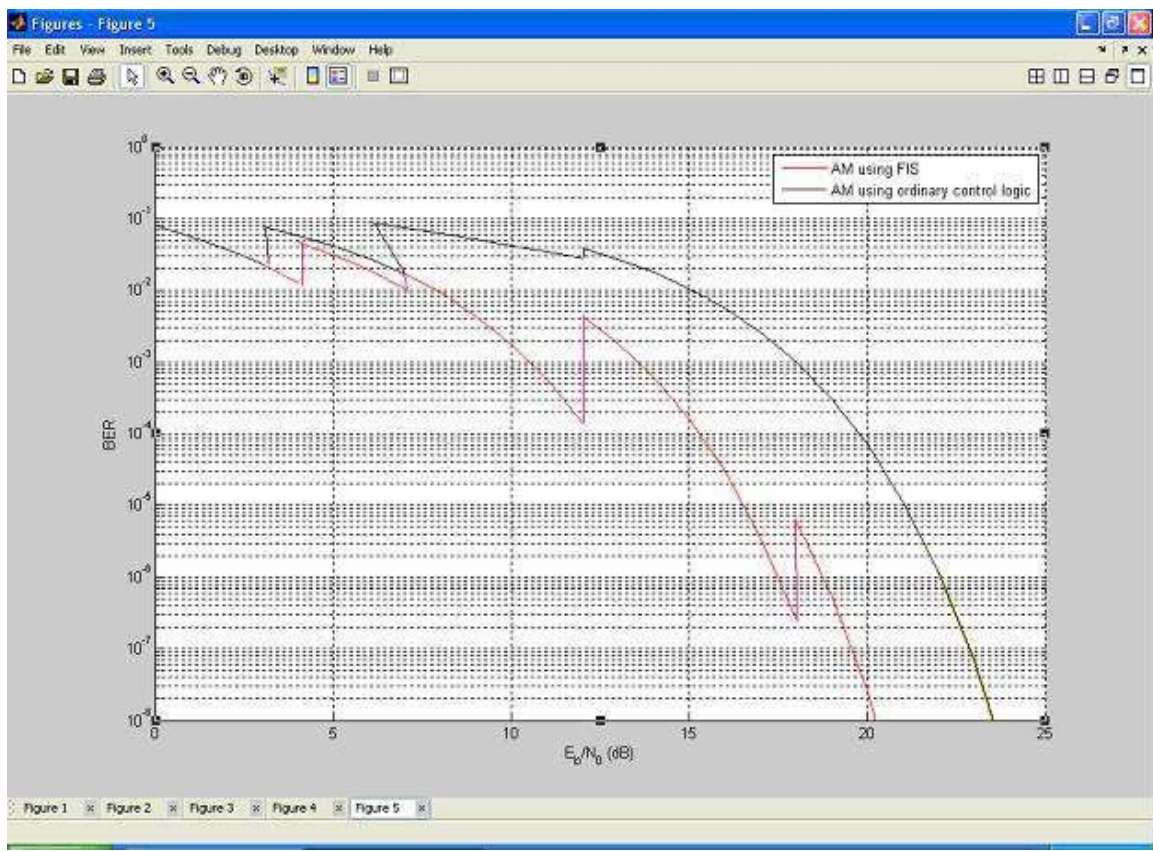

Fig. 18. Comparing Adaptive modulation schemes using FIS and using ordinary control logic 


\section{Conclusion}

Adaptive modulation is a powerful technique for maximizing the data throughput of subcarriers allocated to a user. Adaptive modulation involves measuring the SNR of each subcarrier in the transmission, then selecting a modulation scheme that will maximize the spectral efficiency, while maintaining an acceptable BER. Fuzzy logic based adaptive modulation performs better ordinary logic based adaptive modulation for OFDM system.

\section{References}

K. Seshadri Sastry and Dr.M.S.Prasad Babu, "Adaptive Modulation for OFDM system using Fuzzy logic interface", july 15-18, 2010, IEEE ICSESS, PP 368-371.

K. Seshadri Sastry and Dr.M.S.Prasad Babu, "Fuzzy logic based Adaptive Modulation Using Non Data Aided SNR Estimation for OFDM system", International Journal of Engineering Science and Technology, Vol. 2(6), 2010, pp 2384-2392

Kwang Yoon Kim, Yoon Hyun Kim and Jin Young Kim "Performance of Multi-user MIMO OFDM System with Adaptive Modulation and coding for wireless communications" Feb 15-18, ICACT 2009 Modulation and Coding for Wireless Communications", feb 15-19, 2009, IEEE ICACT 2009

Li Yanxin, Hu Aiqun, "An Adaptive Demodulation Method for QAM Signals" IEEE 2007 International Symposium on Microwave, Antenna, Propagation, and EMC Technologies For Wireless Communications

K.Seshadri Sastry and Dr.M.S.Prasad Babu ", SNR Estimation for QAM signals Using Fuzzy Logic Interface", IEEE ICCSIT 2010, July 2010, pp 413- 416

Jind-Yeh Lee, Huan-Chang and Henry Samueli, "A Digital Adaptive Beamforming QAM Demodulator IC for High Bit-Rate Wireless Communications", IEEE Journal of solid - state circuits, vol. 33, NO. 3, Mar 1998, pp 367-377.

Jouko Vankka, Marko Kosunen, Ignacio Sanchis, and Kari A. I. Halonen, “A Multicarrier QAM Modulator", IEEE Transactions on circuits and systems - II:Analog and digital signal processing, vol. 47, No. 1, jan 2000,pp 1-10.

Franc,ois-Xavier Socheleau, Abdeldjalil A“issa-El-Bey, and S'ebastien Houcke, " Non DataAided SNR Estimation of OFDM Signals", IEEE Communication Letters, VOL. 12, NO. 11, NOV 2008, pp 813-815

K. Seshadri Sastry and Dr.M.S.Prasad Babu," AI based Digital Companding Scheme for OFDM system using custom constellation Mapping and selection", International Journal on Computer Science and Engineering , Vol. 02, No. 04, 2010, pp 1381-1386

Li Yanxin, Hu Aiqun, "An Adaptive Demodulation Method for QAM Signals" IEEE 2007 International Symposium on Microwave, Antenna, Propagation, and EMC Technologies For Wireless Communications

K. Seshadri Sastry and Dr.M.S.Prasad Babu," AI Based Digital Companding scheme for Software Defined Radio" IEEE ICSESS 2010, pp 417- 419

Kiyoshi Hamaguchi, Yukiyoshi Kamio and Eimatsu Moriyama, "Implementation and Performance of an Adaptive QAM Modulation level-controlled System for Land Mobile Communications"

Falahati, Arne Svensson, Torbjörn Ekman and Mikael Sternad, "Adaptive Modulation Systems for Predictive Wireless Channels" 
K. Seshadri Sastry and Dr.M.S.Prasad Babu," Digital Companding Scheme using A I based custom constellation Mapping and selection

K. Pahlavan and J. L. Holsinger, "A Model for the Effects of PCM Compandors on the Performance of High Speed Modems," GLOBECOM '85, New Orleans, December 1985, pp. 24.8.1-24.8.5

M. K. Wasantha and W. A. C. Fernando, "QAM based Adaptive Modulation for OFDMCDMA Wireless Networks",

Li Yanxin, Hu Aiqun, "An Adaptive Demodulation Method for QAM Signals" IEEE 2007 International Symposium on Microwave, Antenna, Propagation, and EMC Technologies For Wireless Communications",

S. Sasaki, J. Zhu and G. Marubayashi, "Performance of parallel combinatory spread spectrum multiple access communication systems," Proceedings of 1991 IEEE International Symposium on Personal, Indoor and Mobile Radio Communications (PIMRC), pp.204-208. 


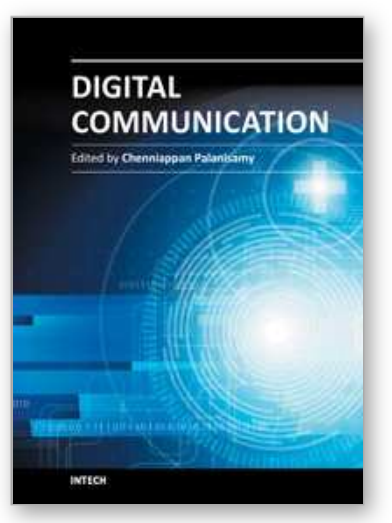

\author{
Digital Communication \\ Edited by Prof. C Palanisamy
}

ISBN 978-953-51-0215-1

Hard cover, 208 pages

Publisher InTech

Published online 07, March, 2012

Published in print edition March, 2012

All marketing is digital and everyone should have a digital strategy. Everything is going mobile. "The world has never been more social" is the recent talk in the community. Digital Communication is the key enabler of that. Digital information tends to be far more resistant to transmit and interpret errors than information symbolized in an analog medium. This accounts for the clarity of digitally-encoded telephone connections, compact audio disks, and much of the enthusiasm in the engineering community for digital communications technology. A contemporary and comprehensive coverage of the field of digital communication, this book explores modern digital communication techniques. The purpose of this book is to extend and update the knowledge of the reader in the dynamically changing field of digital communication.

\title{
How to reference
}

In order to correctly reference this scholarly work, feel free to copy and paste the following:

Seshadri K. Sastry (2012). Adaptive Modulation for OFDM System Using Fuzzy Logic Interface, Digital Communication, Prof. C Palanisamy (Ed.), ISBN: 978-953-51-0215-1, InTech, Available from: http://www.intechopen.com/books/digital-communication/adaptive-modulation-for-ofdm-system-using-fuzzylogic-interface

\section{INTECH}

open science | open minds

\section{InTech Europe}

University Campus STeP Ri Slavka Krautzeka 83/A 51000 Rijeka, Croatia Phone: +385 (51) 770447 Fax: +385 (51) 686166 www.intechopen.com

\section{InTech China}

Unit 405, Office Block, Hotel Equatorial Shanghai

No.65, Yan An Road (West), Shanghai, 200040, China 中国上海市延安西路65号上海国际贵都大饭店办公楼 405 单元

Phone: +86-21-62489820

Fax: +86-21-62489821 
(C) 2012 The Author(s). Licensee IntechOpen. This is an open access article distributed under the terms of the Creative Commons Attribution 3.0 License, which permits unrestricted use, distribution, and reproduction in any medium, provided the original work is properly cited. 